Chapman University

Chapman University Digital Commons

$10-31-2018$

PaU: Diseño de un exergame en una superficie elástica para niños con autismo severo

Gloria I. Monarca

Arturo Morales-Téllez

Franceli L. Cibrian

Mónica Tentori 


\section{PaU: Diseño de un exergame en una superficie elástica para niños con autismo severo}

\section{Comments}

This article was originally published in Avances en Interacción Humano-Computadora, volume 1, in 2018. https://doi.org/10.47756/aihc.y3i1.39

This article is in Spanish.

\section{Creative Commons License}

(C) $(\mathbb{\theta} \Theta \Theta$

This work is licensed under a Creative Commons Attribution-Noncommercial-No Derivative Works 4.0 License. 
TRABAJO EN PROGRESO

\title{
PaU: Diseño de un exergame en una superficie elástica para niños con autismo severo
}

\author{
Gloria I. Monarca, Arturo Morales-Téllez, Franceli \\ L. Cibrian, Mónica Tentori
}

Publicado: 31 Octubre 2018

\begin{abstract}
Resumen
Los niños con autismo severo tienen dificultades para controlar su fuerza al empujar objetos. Existe evidencia de que la repetición de movimientos de empuje usando diferentes niveles de fuerza pudiera mejorar el control de fuerza de niños con autismo. En este artículo se presentan el diseño de $\mathrm{PaU}$ (Push and Up) -un exergame en una superficie elástica para la práctica de movimientos de empuje de niños con autismo severo. Para diseñar PaU se siguió una metodología de diseño centrada en el usuario. Reflexionando en el proceso de diseño se propusieron tres implicaciones de diseño que pudieran ser de utilidad para otros investigadores interesados en diseñar exergames y superficies elásticas para niños con autismo severo. Se cierra este artículo discutiendo el trabajo a futuro y conclusiones.
\end{abstract}

Palabras clave: Exergames, superficies elásticas, autismo, control de fuerza.

\section{Introducción}

El $80 \%$ de niños con autismo severo tienen problemas para controlar su fuerza cuando realizan movimientos, [6,8], es decir, frecuentemente los niños con autismo usan una cantidad insuficiente o excesiva de su fuerza al manipular objetos. Por ejemplo, algunos niños con autismo severo tienen dificultad para controlar la fuerza y dirección cuando empujan un balón [8], o usan más fuerza al interactuar con una tableta que los niños neurotípicos [1]. El control de la fuerza es importante para realizar tareas de la vida diaria (ej., escribir, jugar, alimentarse). Por lo general, para mejorar el control de fuerza es necesario realizar terapia física con un fisioterapeuta. Durante las sesiones de terapia física, existen diferentes ejercicios que pudieran ayudar en el control de fuerza, por ejemplo, empujar una pelota [5]. La literatura de cómputo ubicuo e Interacción Humano Computadora (IHC) ha mostrado que los juegos serios basados en movimiento o exergames son herramientas adecuadas para apoyar las terapias físicas de niños con autismo severo [3]. En particular, esta literatura ha mostrado que las superficies elásticas son eficaces para captar la atención de los niños con autismo y los motiva a

Monarca, GI., Morales A., Cibrian FL., Tentori M.

CICESE

Ensenada, Baja California.

monarca@ cicese.edu.mx, amorales@ cicese.edu.mx,

franceli@cicese.edu.mx,mtentori@cicese.mx realizar movimientos de empuje [4]. Sin embargo, hasta nuestro conocimiento, no se ha explorado como debería ser el diseño de exergames para superficies elásticas que apoyen la terapia física enfocada en mejorar el control de fuerza de niños con autismo severo. En este artículo se presenta el proceso de diseño de un exergame en una superficie elástica para promover el movimiento de empuje de niños con autismo severo ${ }^{1}$. La pregunta de investigación que guía este estudio es: ¿Qué características de diseño debe tener un exergame en una superficie elástica para promover repeticiones del movimiento de empuje de niños con autismo?

\section{Trabajo relacionado}

En esta sección primero se describe el trabajo relacionado con respecto al uso de exergames para niños con autismo. Posteriormente se describe el diseño de superficies elásticas.

\subsection{Exergames para niños con autismo}

Pocos estudios se han enfocado en el diseño de tecnología para ayudar a los niños con autismo a mejorar sus habilidades motoras $[3,4]$. Un ejemplo es, FroggyBobby [3], un exergame que permite a los niños con autismo practicar ejercicios de coordinación visiomotriz. El objetivo del juego es atrapar objetos virtuales con la lengua de una rana que es controlada por los movimientos de los brazos. El uso de FroggyBobby, por siete niños con autismo durante siete semanas, muestra que es una herramienta efectiva para mantener la atención de niños con autismo y ayudarlos a desarrollar movimientos dirigidos de sus extremidades. En general, existe evidencia de que el uso de exergames puede mejorar las habilidades motrices de los niños con autismo. Sin embargo ninguno de estos trabajos promueve la repetición de movimientos de empuje que les pudiera permitir a los niños con autismo mejorar el control de su fuerza.

\subsection{Superficies elásticas}

Pocos trabajos en IHC y Cómputo ubicuo han propuesto superficies elásticas para niños con autismo. Las superficies elásticas son superficies táctiles donde el usuario puede empujar, jalar o estirar una membrana elástica para interactuar con el sistema creando deformaciones temporales [9]. Por ejemplo, DepthTouch [7] es una superficie elástica que proyecta imágenes

\footnotetext{
${ }^{1}$ Por simplicidad de la lectura de aquí en adelante nos referiremos a niños con autismo severo como niños con autismo
} 
Tabla 1 Resumen de las sesiones de diseño

\begin{tabular}{|l|l|l|l|}
\hline & \multicolumn{1}{|c|}{ Objetivo } & \multicolumn{1}{c|}{ Resultado } & \multicolumn{1}{c|}{$\begin{array}{c}\text { Rol (\# de } \\
\text { participantes) }\end{array}$} \\
\hline $\mathbf{1}$ & $\begin{array}{l}\text { Proponer visiones } \\
\text { con respecto a los } \\
\text { temas emergentes. } \\
\text { Analizar su } \\
\text { factibilidad. }\end{array}$ & $\begin{array}{l}\text { 2 visiones de } \\
\text { posibles prototipos. } \\
\text { Análisis de sus } \\
\text { ventajas y } \\
\text { desventajas }\end{array}$ & $\begin{array}{l}\text { Expertos en IHC y } \\
\text { Ubicomp (6) }\end{array}$ \\
\hline $\mathbf{2}$ & $\begin{array}{l}\text { Analizar las } \\
\text { visiones creadas. } \\
\text { Proponer nuevas } \\
\text { visiones. Analizar } \\
\text { ventajas y } \\
\text { desventajas }\end{array}$ & $\begin{array}{l}\text { Una visión para un } \\
\text { exergame Análisis } \\
\text { de la factibilidad de } \\
\text { las tres visiones }\end{array}$ & $\begin{array}{l}\text { Expertos en IHC y } \\
\text { Ubicomp (4), } \\
\text { Psicóloga (2) }\end{array}$ \\
\hline $\mathbf{3}$ & $\begin{array}{l}\text { Elegir una visión } \\
\text { para desarrollar el } \\
\text { guion gráfico. }\end{array}$ & $\begin{array}{l}\text { Elección de una } \\
\text { visión } \\
\text { Especificación del } \\
\text { guion gráfico }\end{array}$ & $\begin{array}{l}\text { Expertos en IHC y } \\
\text { Ubicomp (3), } \\
\text { Psicóloga (1) } \\
\text { Músico(1) }\end{array}$ \\
\hline
\end{tabular}

de esferas sobre la superficie. Las esferas imitan las fuerzas gravitacionales según la fuerza aplicada cuando los usuarios empujan la superficie. El uso de DepthTouch muestra que en una superficie elástica es fácil de entender cómo funcionan las interacciones relacionadas con la fuerza.

Otro ejemplo es BendableSound [4], una superficie multisensorial elástica que permite a los niños con autismo crear música y practicar patrones de movimiento. Un estudio con 24 niños con autismo muestra que BendableSound mantiene la atención de los niños y los motiva a realizar movimientos de empuje y deslizamiento con una mano, dos manos, e incluso usando diferentes partes del cuerpo. A pesar de que los trabajos mencionados muestran que los exergames tienen un impacto en la motricidad de los niños con autismo y las superficies elásticas motivan la realización de movimientos de empuje, hasta nuestro conocimiento no se ha explorado como se debe diseñar un exergame para promover las repeticiones de movimientos de empuje en superficies elásticas. En la luz de esta literatura las contribuciones de este artículo son: (1) descripción del proceso de diseño para un exergame en una superficie elástica para niños con autismo; (2) cuatro implicaciones para el diseño de un exergame en una superficie elástica para niños con autismo; (3) descripción de $\mathrm{PaU}$, ejemplo de un exergame en una superficie elástica donde se aplicaron los principios de diseño.

\section{Métodos}

Por 2 meses se siguió una metodología cualitativa de diseño centrada en el usuario para desarrollar un exergame en una superficie elástica. Durante el estudio se recolectó un banco de 10 entrevistas semi-estructuradas de trabajos previos relevantes realizadas a psicólogos, fisioterapeutas, y cuidadoras de la clínica Pasitos [4]. Los temas que se preguntaron en las entrevistas involucran las preferencias de los niños con autismo, como se realizan las terapias físicas para el control de fuerza, a que problemas se enfrentan los terapeutas y psicólogos durante las terapias y que estrategias siguen para resolverlos (Duración total = 10 horas; $\mu=53.10 \mathrm{~min}$ y $\mathrm{s}=12.37 \mathrm{~min}$ ). Las entrevistas se analizaron de manera colaborativa en cuatro sesiones de interpretación con una duración total de 5 horas aproximadamente ( $\mu=83.10 \mathrm{~min}$ y $\mathrm{S}=1.87 \mathrm{~min}$ ). Se utilizaron técnicas de diseño contextual rápido para encontrar los temas emergentes y crear un diagrama de afinidad. Para involucrar de manera activa a los especialistas en el proceso de diseño, se complementaron las entrevistas con 3 sesiones de diseño. En estas sesiones participaron expertos en IHC, psicología y música (Tabla 1, duración total $=6$ horas, $\mu=115.10 \mathrm{~min}$ y $S=2.37 \mathrm{~min})$. La dinámica de las sesiones fue la siguiente: primero se les explicaba a los participantes el contexto del estudio; posteriormente se realizaron lluvia de ideas y se discutieron potenciales ideas de diseño; finalmente, se propusieron prototipos de baja fidelidad y se discutieron ventajas y desventajas de los prototipos. Los datos recolectados durante las sesiones de diseño fueron analizados y se materializaron como bosquejos (sketches) y guiones gráficos (storyboards).

\section{Resultados}

Los resultados del artículo se han dividido en tres: las implicaciones de diseño encontradas, las visiones iniciales y el diseño de $\mathrm{PaU}$ (Push and Up) - un exergame en una superficie elástica para la práctica de movimientos de empuje de niños con autismo.

\subsection{Implicaciones de diseño.}

Como resultado de las entrevistas, y sesiones de diseño se obtuvieron las siguientes implicaciones requeridas para desarrollar un exergame que promueva el movimiento de empuje en una superficie elástica.

- Utilizar reforzadores positivos: los exergame en superficies elásticas deben desarrollar un modelo de recompensas y retroalimentación que incentive a los niños con autismo a utilizar la superficie y realizar la repetición de movimientos. Los especialistas sugirieron utilizar sonidos como retroalimentación de los movimientos y animaciones como recompensas.

- Utilizar estímulos sensoriales atractivos: las superficies elásticas deben agregar visualizaciones y sonidos lo suficientemente atractivos para ayudar a mantener la atención de los niños con autismo mientras obtienen retroalimentación táctil de su fuerza. Por ejemplo, los especialistas sugirieron usar imágenes tipo caricatura y elementos coloridos como estímulo visual, y sonidos relacionados a las visualizaciones (ej. usar sonido de corrientes de viento si el escenario es en el cielo). Esto ayuda a captar la atención de los niños con autismo y mantenerlos enganchados.

- Utilizar instrucciones claras y sencillas: Para evitar la frustración de los niños con autismo al usar un exergame en una superficie elástica, se sugiere que el exergame integre instrucciones simples y claras. Los especialistas sugirieron que los exergames primeramente deben proveer una instrucción verbal, es decir, el exergame debe decir "Empuja"). En caso de no obtener la atención del niño, el exergame entonces debe proveer una instrucción modelada, es decir, se debe presentar una animación visual que acompañe a la instrucción y le muestre al niño que es lo que tiene que hacer. Es importante evitar la frustración para que los niños con autismo quieran seguir jugando.

- Usar analogías para motivar el movimiento de empuje con diferentes niveles de fuerza. Durante las terapias, los niños deben explorar diferentes niveles de fuerza usando una o ambas manos. Por lo que los especialistas sugirieron usar analogías de catapultar un objeto para obtener recompensas que estén cerca o lejos de acuerdo a la fuerza aplicada, i.e., si los niños empujan con mayor fuerza el objeto a catapultar llegara más lejos. 


\subsection{Visiones iniciales del diseño}

Basados en las implicaciones de diseño se obtuvieron 3 prototipos de baja fidelidad: empujar un globo aerostático, limpiar la superficie elástica y empujar balones (ver Tabla 2). Dado que el prototipo de empujar el globo aerostático promueve la repetición de movimientos de empuje, tiene visualizaciones atractivas y se puede generalizar a otros escenarios, los especialistas seleccionaron esta alternativa como la más apropiada para un exergame en una superficie elástica.

\subsection{PaU: Un exergame en una superficie elástica para niños con autismo severo}

$\mathrm{PaU}$ (Push and Up) es una superficie elástica para promover la repetición del movimiento de empuje. El juego se proyecta en una superficie elástica y los niños con autismo deben empujar dicha superficie para jugar (Figura 1). PaU cuenta con tres niveles: agua, tierra, espacio (Figura 2).

\subsubsection{Uso de estímulos sensoriales atractivos}

La visualización de cada nivel de PaU está compuesta por los siguientes elementos (Figura 3): escenario, avatar, barra de progreso, objetos a recolectar y unas manos que sirven como guía. Todos los elementos tienen una forma caricaturista y colorida. El fondo de cada escenario cambia según el nivel (mar, cielo, espacio). El avatar es un personaje animado que va dentro de un globo aerostático, submarino o cohete, según el nivel en el que se encuentre. Como estímulo auditivo al realizar el movimiento de empuje se escuchara el sonido correspondiente al escenario (i.e., agua, viento, canción de estrellita).

\subsubsection{Uso de reforzadores positivos}

Para mantener la atención de los niños con autismo, en cada nivel de $\mathrm{PaU}$ aparecen objetos que el avatar debe ir recolectando cuando el niño empuje la superficie elástica. Si los objetos aparecen cerca, los niños con autismo deben empujar de forma suave la superficie. Por el contrario, si los objetos aparecen lejos, los niños con autismo deben empujar fuerte. Al recolectar los objetos la barra de progreso va aumentando. Al llenar la barra de progreso, se despliega la visualización de la recompensa. La recompensa corresponde a un video de alguna caricatura. Después de obtener la recompensa inicia el siguiente nivel.

\subsubsection{Uso de instrucciones claras para evitar frustración}

Para no frustrar a los niños con autismo mientas juegan con $\mathrm{PaU}$, el avatar nunca retrocede. En caso de que no se detecte alguna interacción del niño con la superficie, el avatar se queda en la misma posición y aparecen las instrucciones. La primera instrucción que se da es verbal, por lo que se escucha la instrucción "Empuja". Posteriormente, si el niño no realiza la actividad se le da la da una instrucción de manera visual mostrando unas manos que dan la ilusión de empujar. En caso que no realice el movimiento, con la instrucción visual, el juego es pausado y aparece el avatar modelando el movimiento de empuje.

\subsubsection{Dinámica del juego}

Primero se selecciona un nivel de manera aleatoria. Una vez seleccionado el nivel, se proyecta en el centro la superficie el avatar y se le pide al niño que empuje para poder avanzar. El objetivo principal de cualquier nivel en $\mathrm{PaU}$ es catapultar al avatar mediante el movimiento de empuje. Para recolectar los objetos y llenar la barra de progreso los niños deben empujar fuerte para recolectar los objetos que aparecen lejos y suave para recolectar
Tabla 2 Descripción de las visiones iniciales de diseño

\begin{tabular}{|c|l|l|l|}
\hline Exergame & \multicolumn{1}{|c|}{ Objetivo } & \multicolumn{1}{c|}{ Ventajas } & Desventajas \\
\hline $\begin{array}{c}\text { Globo } \\
\text { aerostático }\end{array}$ & $\begin{array}{l}\text { Utilizar } \\
\text { diferentes } \\
\text { niveles de } \\
\text { fuerza para } \\
\text { que el globo } \\
\text { avance }\end{array}$ & $\begin{array}{l}\text { Promueve } \\
\text { repeticiones y } \\
\text { control de fuerza. } \\
\text { Visualizaciones } \\
\text { atractivas } \\
\text { Se puede generalizar } \\
\text { a otros escenarios }\end{array}$ & $\begin{array}{l}\text { Área } \\
\text { restringida }\end{array}$ \\
\hline $\begin{array}{c}\text { Limpiar } \\
\text { superficie }\end{array}$ & $\begin{array}{l}\text { Empujar para } \\
\text { limpiar una } \\
\text { superficie }\end{array}$ & $\begin{array}{l}\text { Promueve } \\
\text { repeticiones del } \\
\text { movimiento de } \\
\text { empuje } \\
\text { Mayor área de } \\
\text { interacción }\end{array}$ & $\begin{array}{l}\text { Se vuelve } \\
\text { aburrido. } \\
\text { No es muy } \\
\text { llamativo }\end{array}$ \\
\hline $\begin{array}{c}\text { Empujar } \\
\text { balones }\end{array}$ & $\begin{array}{l}\text { Empujar } \\
\text { balones para } \\
\text { tirar conos }\end{array}$ & $\begin{array}{l}\text { Promueve } \\
\text { repeticiones del } \\
\text { movimiento de } \\
\text { empuje. } \\
\text { Motiva practicar } \\
\text { deportes }\end{array}$ & $\begin{array}{l}\text { Motiva } \\
\text { movimientos } \\
\text { cortos. }\end{array}$ \\
& & $\begin{array}{l}\text { Área } \\
\text { restringida }\end{array}$ \\
\hline
\end{tabular}

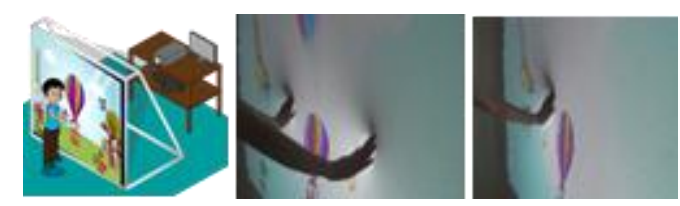

Figura 1 Configuración de PaU en una superficie elástica (izquierda). Un usuario empujando la superficie elástica con las dos manos (centro), y usando la mano izquierda (derecha) para que el globo recolecte los objetos que aparecen en el escenario.

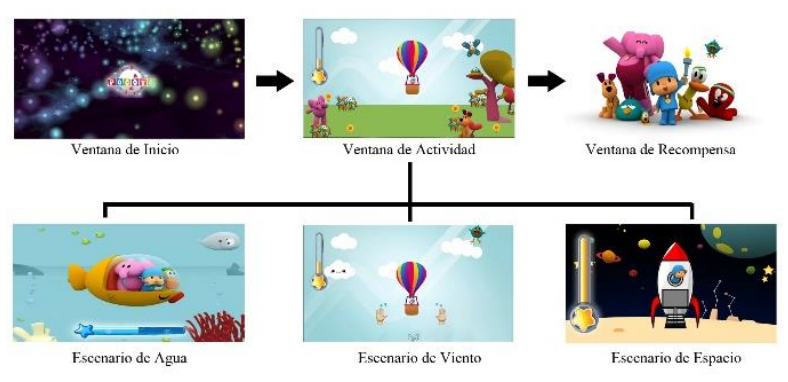

Figura 2. Esquema que representa la dinámica del juego de PaU

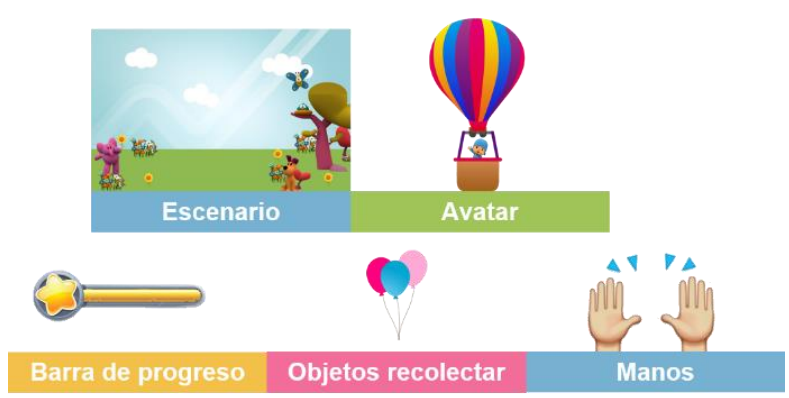

Figura 3. Elementos que componen PaU 
los objetos que aparecen más cerca. Cada que el niño empuja se escucha un sonido relacionado a la temática. Una vez que se ha llenado la barra de progreso, aparece la ventana de recompensa, donde se me muestra un segmento de caricatura. Al finalizar, la caricatura empieza el siguiente nivel. Una vez completados los tres niveles se le muestra un episodio completo de una caricatura.

\subsubsection{Escenario de uso}

Para ejemplificar como se utiliza PaU en la práctica se presenta el siguiente escenario:

Panchito es un niño de 6 años de edad con autismo severo que presenta dificultades al controlar su fuerza. Hoy la profesora Lucy lleva a Panchito a la sala donde está instalado PaU. Panchito al entrar a la sala se percata inmediatamente de $\mathrm{PaU}$ por su interfaz brillante. Panchito se acerca y presiona la tela por primera vez, entonces $\mathrm{PaU}$ muestra el escenario del agua y Panchito escucha la palabra “¡Empuja!”. Panchito empuja la tela y escucha un sonido de agua mientras observa como el avatar empieza a subir. Panchito realiza el movimiento múltiples veces para avanzar y recolectar diferentes objetos. Al ir recolectando objetos, la barra de progreso empieza a aumentar. Cuando la barra de progreso se completa, PAU muestra una caricatura de Pocoyo como recompensa. Panchito se emociona y comienza a reír. La profesora Lucy felicita a Panchito por haber conseguido la recompensa final

\section{Conclusiones y trabajo futuro}

En este trabajo presentamos un estudio siguiendo la metodología de diseño centrada en el usuario para diseñar $\mathrm{PaU}$ - un exergame en una superficie elástica para la práctica de movimientos de empuje de niños con autismo severo. En este estudio aprendimos que los exergame en superficies elásticas para niños con autismo deben de utilizar reforzadores positivos, estímulos sensoriales atractivos, evitar la frustración y motivar el uso de diferentes niveles de fuerza. Para completar el proceso de diseño se propone como trabajo futuro: (1) Implementar las actividades de PaU (2) Realizar un estudio formativo del uso de $\mathrm{PaU}$ para investigar si las actividades y el diseño son adecuados (3) Realizar un estudio sumativo para evaluar el impacto de $\mathrm{PaU}$ en la regulación de la fuerza de niños con autismo.

\section{Agradecimientos}

Agradecemos a todos los participantes en este estudio y a CONACYT.

\section{Referencias}

[1] A. Anzulewicz, K. Sobota, and J. T. Delafield-butt, "Toward the Autism Motor Signature : Gesture patterns during smart tablet gameplay identify children with autism," Nat. Publ. Gr., no. August, pp. 1-13, 2016.

[2] A. Bhattacharya, M. Gelsomini, P. Pérez-Fuster, G. D. Abowd, and A. Rozga, "Designing motion-based activities to engage students with autism in classroom settings," in Proceedings of the 14th International Conference on Interaction Design and Children - IDC '15, 2015, pp. 69-78.

[3] K. Caro, M. Tentori, A. I. Martinez-Garcia, and I. ZavalaIbarra, "FroggyBobby: An exergame to support children with motor problems practicing motor coordination exercises during therapeutic interventions," Comput. Human Behav., vol. 71, pp. 479-498, Jun. 2017.

[4] F. L. Cibrian, O. Peña, D. Ortega, and M. Tentori, "BendableSound: An elastic multisensory surface using touch-based interactions to assist children with severe autism during music therapy," Int. J. Hum. Comput. Stud., vol. 107, pp. 22-37, Nov. 2017.

[5] "Examples of outpatient physical therapy activities the importance of data collection in measuring progress.'[Online].Available:

https://www.carautismroadmap.org/examples-of-outpatientphysical-therapy-activities/?print=pdf. [Accessed: 15-May2018].

[6] K. A. Fournier, C. J. Hass, S. K. Naik, N. Lodha, and J. H. Cauraugh, "Motor Coordination in Autism Spectrum Disorders: A Synthesis and Meta-Analysis," J. Autism Dev. Disord., vol. 40, no. 10, pp. 1227-1240, Oct. 2010.

[7] M. Müller, T. Gründer, and R. Groh, "Data exploration on elastic displays using physical metaphors," xCoAx 2015 Proc. Third Conf. Comput. Commun. Aesthet. X, pp. 111124, 2015.

[8] K. L. Staples and G. Reid, "Fundamental Movement Skills and Autism Spectrum Disorders," J. Autism Dev. Disord., vol. 40, no. 2, pp. 209-217, Feb. 2010.

[9] G. M. Troiano, E. W. Pedersen, and K. Hornbaek, "UserDefined Gestures for Elastic, Deformable Displays." 